\title{
Phenotypic Similarities Within The Morphologic Spectrum Of DICER1-Associated Sarcomas and Pleuropulmonary Blastoma - Histopathologic Features Suggesting Genetic Testing and Counselling
}

\author{
Paromita Roy ${ }^{1}$, Anirban Das ${ }^{1}$, Angad Singh ${ }^{1}$, Joyshree Panda ${ }^{1}$, Arpita Bhattacharya ${ }^{1}$, \\ Anisha Gehani ${ }^{1}$, Mayur Parihar ${ }^{1}$, Reghu Sukumaran ${ }^{1}$, Rimpa Achari ${ }^{1}$, Rita Alaggio ${ }^{2}$, \\ Amanda Field ${ }^{3}$, Ashley Hill ${ }^{4}$, Louis Dehner ${ }^{5}$, and Kris Ann Schultz ${ }^{6}$ \\ ${ }^{1}$ Tata Medical Center \\ ${ }^{2}$ University of Padova \\ ${ }^{3}$ ResourcePath \\ ${ }^{4}$ George Washington University School of Health Sciences \\ ${ }^{5}$ Washington University in Saint Louis \\ ${ }^{6}$ University of Minnesota
}

June 7, 2021

\begin{abstract}
Context - DICER1-associated sarcomas are rare. These are currently described under a wide variety of appellations; morphologic characterizations in reported cases and sites of occurrence have also been disparate. Design - We aimed to review pediatric sarcomas associated with DICER1-mutation reported at our center, along with literature review, to identify histologic hallmarks for diagnosis. Results - A 12 year old girl with intracranial sarcoma mimicking mesenchymal chondrosarcoma, a 16 year old girl with broad ligament sarcoma mimicking fibrosarcoma and a 5 month old girl with vaginal sarcoma mimicking embryonal rhabdomyosarcoma showed DICER1-mutation. All three tumors though seemingly diverse, had an uncanny resemblance, comprising of a primitive mesenchyme-like spindle cell component with rhabdomyoblastic differentiation on immunohistochemistry. Primitive blastema, chondroid differentiation and foci of anaplasia mimicking pleuropulmonary blastoma histology were variably present. One case showed primitive neuroblastic differentiation. Though the constellation of features reported in literature is quite varied, rhabdomyoblastic differentiation has been ubiquitously reported in tumours across sites. Molecular testing showed gain of chromosome 8 in 2 cases. All 3 cases responded to alternating Vincristine, doxorubicin and cyclophosphamide / Cisplatin, etoposide and ifosfamide (VDC/PEI) backbone followed by maintenance chemotherapy. Conclusion - We highlight this morphologic hallmark of rhabdomyoblastic differentiation with or without chondroid differentiation, in primitive appearing pediatric sarcomas, especially of female genital tract and brain, which should raise a flag to test for DICER1 pathogenic variation. This is crucial in low / middle income countries where sequencing is not done routinely. Timely diagnosis can ensure appropriate treatment and implementation of surveillance protocols for those with germline mutation.
\end{abstract}

\section{Introduction :}

The DICER1 -tumor predisposition syndrome (OMIM 6012000) is an autosomal dominant disorder with incomplete penetrance and exhibits multiple tumors over time in affected kindreds. ${ }^{1-6}$ It is characterized by heterozygous germline mutation in DICER1 gene. ${ }^{4}$ DICER 1 -associated tumors can also be due to biallelic somatic mutations and can be grouped into sarcomas and non-sarcomatous tumors. These comprise a 
varied spectrum of unrelated benign to malignant tumours, as well as non-neoplastic entities. ${ }^{7-9}$ DICER1 -associated sarcomas have been reported in literature under a varied list of appellations such as pleuropulmonary blastoma (PPB; the prototype), embryonal rhabdomyosarcoma (ERMS), anaplastic sarcoma of kidney, adenosarcoma, primitive intracranial sarcoma, carcinosarcoma, fibrosarcoma, leiomyosarcoma, primitive neuroectodermal tumour (PNET), mesenchymal chondrosarcoma and more recently PPB-like peritoneal sarcoma..$^{7-9}$ It is increasingly being recognized that these sarcomas may be morphologically similar enough for a unified terminology - DICER 1 -associated sarcoma. ${ }^{7,10}$

We present a series of three DICER1 -associated extrapulmonary sarcomas, each of whom presented with a spindle cell sarcoma of primitive morphology and rhabdomyoblastic differentiation on immunohistochemistry and did not respond to conventional rhabdomyosarcoma chemotherapy. We compared the morphology with that of other reported DICER1 -associated sarcomas to identify morphologic clues to guide appropriate molecular testing for diagnosis of these tumours.

\section{Methods :}

Three pediatric extrapulmonary spindle cell sarcomas with primitive morphology and rhabdomyosarcomatous differentiation which raised suspicion for association with DICER1 pathogenic variation were studied. The cases were enrolled in the International Pleuropulmonary Blastoma / (PPB) / DICER1 Registry. Institutional review board approval was sought (EC/WV/TMC/41/20). Tumour sequencing was done at Heidelberg Institute, on Illumina NextSeq platform for 130 brain tumour related genes applying panel NPHD2015A, enriched with Agilent SureSelect method for 1 case. This was followed by confirmation of germline alteration using Sanger sequencing. DNA methylation profiling was also done with Illumina Human Methylation 850 (850k) array and internal classified V11b4 and sarcoma_v6.3. For other two cases sequencing was performed using the Ion Torrent Personal Genome Machine (PGM) for coding regions of DICER1, p53 and a single amplicon of FOXL2, at ResourcePath, through the International PPB/DICER1 Registry and Children's Minnesota, Minneapolis, MN.

All immunohistochemical studies on the cases were done in-house, on the Leica Bondmax. Fluorescent insitu hybridization (FISH) evaluation for chromosome 8 using MYC/CEN-8 dual colour probe (Zytovision $\mathrm{GmbH}$, Germany) was performed in 2 cases.

Clinical details were retrieved from the hospital electronic medical records.

\section{Results :}

We present here the clinicopathologic details of the 3 cases (see Table 1 for summary). Biallelic mutations in the DICER 1 gene on exon 11, was detected in all 3 tumors. Germline testing was possible only in 1 case.

\section{Clinical features}

\section{Case 1}

A 12-year-old female presented with headache, vomiting, left hemiparesis and an episode of generalized seizure. Magnetic resonance imaging (MRI) brain (Figure 1a) showed a heterogeneous lesion with postcontrast enhancement in the right fronto-parietal para-falcine region with surrounding edema and midline shift. Imaging revealed no additional findings. History of an aunt who died of recurrent gynecologic malignancy at 39 years was noted. After gross total resection, radiation therapy (59.4Gy in 33 fractions) was administered. Follow-up chest computed tomography $(\mathrm{CT})$ scans showed multiple, small pulmonary nodules $(<4 \mathrm{~mm})$. Adjuvant chemotherapy with vincristine, doxorubicin and cyclophosphamide (VDC) alternating with cisplatin, etoposide and ifosfamide (PEI) was administered for 10 weeks, followed by vincristine, dactinomycin and cyclophosphamide (VAC) upto 45 weeks. Magnetic resonance imaging (MRI) brain revealed no tumour and positron emission tomography (PET) scan showed few calcified FDG non-avid nodules at the end of therapy. The child's current surveillance protocol includes 6 monthly CT chest and ultrasound (USG) pelvis and 2 yearly USG thyroid. She remains in remission after 3 years.

Case 2 
A 16-year-old girl presented with abdominal pain and decreased appetite. An emergency laparotomy revealed a necrotic mass based in the broad ligament, involving both adnexae, pouch of Douglas with extensive omental and peritoneal deposits (Fig. 1b). Residual tumour responded well to adjuvant chemotherapy with VDC alternating with PEI (6 cycles) (Fig. 1c). Following chemotherapy, a debulking procedure included left salpingo-oophorectomy, total omentectomy and excision of peritoneal deposits. The patient is on maintenance chemotherapy with vinorelbine and cyclophosphamide and is doing well 6 months postsurgery. No significant family history was noted.

Case 3

A five-month-old female presented with a mass protruding into vaginal introitus. Imaging revealed no other lesion. The initial biopsy was reported as an embryonal rhabdomyosarcoma.

The patient was treated with chemotherapy as per the RMS 2005 EpSSG protocol including ifosfamide, vincristine and dactinomycin. Interim assessment after 4 months suggested suboptimal response. She received second line chemotherapy including doxorubicin and carboplatin for 6 months. Reassessment MRI showed a small vaginal nodule with fibrotic signal (Fig. 1d). The parents refused local treatment with radiotherapy and she was followed with imaging-based surveillance. After 6 months a $2 \mathrm{~cm}$ mass extruding into vagina was seen. Repeat scans showed a large recurrent tumour (Fig. 1e, f) with retroperitoneal lymph nodes and mural nodules in the colon. Chemotherapy was commenced with vincristine, irinotecan and temozolomide, however further progression prompted initiation of salvage chemotherapy with PEI alternating with VDC. Despite good response, the parents refused further surgery or radiotherapy. Family history was not contributory, and the family did not consent to germline testing. She had massive progression of disease and was lost to follow-up 2.5 years from presentation.

Pathologic findings :

Case 1

Both resections showed sheets of small round to spindled primitive blastemal cells, with condensed chromatin and small nucleoli. Nodules of immature cartilage were seen to arise abruptly, in the midst of blastemal cells (Fig. 2a-f). Some foci showed alternating hyper and hypocellular spindle cells foci with larger vesicular nuclei and prominent nucleoli (Fig. 2d). Brisk mitoses and apoptosis is noted in both blastemal and sarcomatous foci.

Immunohistochemistry (IHC) with desmin, myogenin, myoD1 and CD99 showed patchy but strong staining in the spindle cell component, while GFAP, smooth muscle actin, synaptophysin and NSE were negative. INI1 was retained in the tumour cells. S100 stained the chondroid elements but was negative in the spindle cell component. Ki 67 proliferation index was $>90 \%$.

\section{Case 2}

Grossly, the tumour was a single solid-cystic mass measuring $10.0 \times 5.5 \mathrm{~cm}$, with multiple irregular, friable projections. On microscopy, alternating sheets and ribbons/trabeculae of densely packed primitive blastemal, monotonous small, round to spindle shaped cells, were separated by hypocellular areas of small spindle cells in collagenized to loose, myxoid stroma (Fig. 3a-f). Areas of perivascular tumour cells, condensation, microcystic foci and cambium-like concentration below pseudocystic spaces were noted (Fig. 3b, 3c). Sarcomatous overgrowth of large spindle cells with abundant clear to eosinophilic cytoplasm, coarse chromatin and prominent nucleoli was present (Fig 3e-f). Brisk mitoses (18-20 per 10 high power fields) including atypical forms and necrosis were identified. Focal immature cartilage was identified in 1 slide (Fig. 3a). The tumour showed a prominent vascular pattern with large dilated blood vessels with thin wall.

Immunohistochemically tumour cells were diffusely positive for desmin and myogenin. There was patchy positivity for myoD1, while AFP, SALL4, CD30, CK, inhibin, PAX8 and WT-1 were negative. INI1 was retained. Cytogenetic studies for PAX-3 or FOXO1 rearrangements were negative.

Case 3 
The initial biopsy of the tumour showed multiple tumour fragments lined by squamous epithelium (Fig. 4af). A largely hypocellular pattern with small, bland spindle or stellate cells resided in a myxoid background with an immature mesenchymal appearance with focal hypercellular areas and perivascular condensation by smaller, angulated cells. These cells also showed subepithelial concentration, forming a cambium-like layer (Fig. 4b). Also noted were larger blastemal cells with coarse nuclear chromatin and prominent nucleoli, arranged in nests, cords and rosette formations, with a granular pink neuropil-like background (Fig. 4e). Sarcomatous areas composed of nests of larger cells with vesicular nuclei, prominent nucleoli and moderate clear cytoplasm, were also noted (Fig. 4f). Mitotic figures were brisk and more frequent in these foci, along with prominent apoptosis. Cartilage was not identified.

On IHC, myoid markers - desmin (Fig. 4b; inset), myogenin, and myoD1 showed patchy positivity in the small, spindle shaped cells, while the larger blastic cells in cords, nests and rosettes were non-reactive. Both components of the tumour are negative for cytokeratin (AE1/AE3), inhibin, SALL4, synaptophysin and CD99.

The second biopsy of the small residual nodule, showed hypocellular tissue with scattered, bland, spindled to stellate cells, in a loose collagenous background. Few of the spindle cells were desmin positive. No blastemal cells were identified in this biopsy. (Fig. 4g)

The third biopsy of the recurrent tumour, revealed sarcomatous overgrowth, comprising of sheets and nests of round to spindle shaped large cells, with coarse chromatin and abundant mitoses and apoptosis. These cells were morphologically similar to the large blastic cells in cords and nests in the initial biopsy but showed greater degree of anaplasia. (Fig. 4h, i) On IHC, the cells showed no rhabdomyoblastic differentiation, being negative for desmin, myoD1 and myogenin. The tumour cells were diffusely positive for CD99 and SALL4 (Figure 3i; inset), focally positive for synaptophysin and neuron specific enolase (NSE). The tumour cells were negative for chromogranin, cytokeratin (AE1/AE3), calretinin, inhibin, S100, Oct3/4, CD45 and Tdt. INI1 was retained. FISH for EWSR1 gene rearrangement was negative.

\section{Discussion :}

As PPB is the archetype of DICER1- associated neoplasms, it is not surprising that several of the extrapulmonary DICER1 mutated sarcomas share aspects of its morphologic template from a cystic to solid macroscopic appearance, to one or more of the histologic patterns. The basic collage consists of a variable intermixture of rhabdomyosarcoma usually embryonal type, islands and nests of blastema, primitive spindle cell pattern with presence of cambium layer, nodules of fetal to sarcomatous cartilage and anaplastic tumor cells. The various sarcomatous patterns are heterogeneously represented, so that blastema is the dominant pattern in one tumor and spindle cells sarcoma in another. This similarity to PPB was noted by Schultz et al and they proposed the designation 'pleuropulmonary blastoma-like peritoneal sarcoma'. 8

Warren et al noted in their literature review that mostDICER1- associated sarcomas had a component of undifferentiated small round blue cells with rhabdomyoblastic differentiation (in 64/71 cases; 90.1\%), poorly differentiated spindle cell component with anaplasia $(45 / 50 ; 90 \%)$ and chondroid/myxoid differentiation $(39 / 45 ; 86.7 \%)$. This similarity was seen in spite of the different nomenclature used for the reported cases and the varied sites of presentation. Though this was not addressed, the morphologic descriptions have a striking resemblance to PPB. Rhabdomyoblastic differentiation was the most common feature in all these cases, (including in the 3 cases reported by them), while the other features were present in variable frequency. Appreciating the commonality in morphology, they proposed a unified terminology for these tumours 'DICER 1 -associated sarcoma', which was further supported by McCluggage et al. 7,10

In our 3 cases, similarities with PPB type III include primitive spindle cell pattern, cambium-like arrangement of tumour cells, nodules of fetal to sarcomatous cartilage and a variable population of anaplastic tumor cells. Chondroid differentiation and anaplastic component was lacking in one case. small focus of immature cartilage was identified in only one out of 11 sections from tumour. Although chondroid differentiation may be focal and could be missed in a biopsy, however, in our experience, when present it is quite specific for DICER1 sarcoma and should prompt testing for DICER1 pathogenic variation. None of the tumors however 
had a neoplastic epithelial component and rhabdomyoblastic differentiation was the only constant feature in all 3 cases. However, this rhabdomyoblastic differentiation was somewhat patchy in all 3 cases, both morphologically and on immunohistochemical staining (with desmin, myogenin and myoD1), which raised our suspicion and helped avoid misclassification as typical rhabdomyosarcoma.

Another morphologic variation not described in the Warren review, is the rare multi-patterned DICER1 -associated tumour with primitive neuroepithelial elements, designated as 'pre-sacral malignant teratoid neoplasm in association with pathogenic DICER1 variation'. ${ }^{9}$ We also noted neuropil with possible focal neuroblastic-like component in Case 3, but in all these tumours and ours, the predominant component was ERMS. Pancaldi et al reported aDICER1 associated PNET with EWSR1 rearrangement which did not show any ERMS component. ${ }^{11} \mathrm{~A}$ unique observation in our case was the positivity of SALL4 and synaptophysin in the recurrent high grade sarcoma, which was negative for myoid markers. Warren and McCluggage have separately reported 2 ovarian tumours, where the small spindle cells were SALL4 positive, but dually positive for myoid markers (desmin and myogenin). ${ }^{7,12}$

The DICER 1 -associated sarcomas seem to have a predilection for the female genital tract and brain. 7,8,12,13 However, rare cases have been reported in males as well, including the para-testicular region. ${ }^{14}$

The PPB-like morphologic spectrum ranging from benign to overtly malignant sarcoma, is also noted in DICER 1 associated renal cystic nephromas and anaplastic sarcoma of kidney, both of which also show rhabdomyoblastic differentiation. ${ }^{7,15}$

Like in cystic nephromas and type $1 \mathrm{PPB}$, the spindle cell morphology in some DICER1 associated tumours can be very bland and hypocellular in some tumours and can be misinterpreted on a small biopsy as fibrotic tissue (as with the recurrent vaginal tumour case 3), even though the cells express RMS markers on IHC. This has also been highlighted by Yoon et al. ${ }^{16}$ Routine use of desmin to evaluate genital tract spindle cell lesions in young females, may help correctly identify these tumours.

The association of rhabdomyosarcomas of the female genital tract withDICER1 has been reported variably in literature, but it is not clear yet if identification of any ERMS element should prompt consideration of a DICER 1 -related neoplasm and justify testing, especially in the absence of immature cartilage or primitive mesenchyme. This question is most pertinent for children where ERMS is known to be one of the commonest childhood sarcomas. While Apellaniz-Ruiz in their review showed that nearly all gynecologic ERMSs (except vaginal tumours) were DICER1 -associated, Doros et al detected DICER1 mutation in only $3.8 \%(2 / 52)$ cases of sporadic ERMS and the Children's oncology group cohort of pediatric rhabdomyosarcomas showed DICER1 germline mutation in $4.4 \%$. ${ }^{10,17-20}$

DICER 1 -associated central nervous system sarcomas also have a high association with rhabdomyosarcomatous differentiation. ${ }^{8,21,22}$ Our CNS case mimicked mesenchymal chondrosarcoma, though it expressed ERMS markers.

We found the presence of gain of chromosome 8 in cases 1 and 2 (FISH was attempted in case 3, but failed quality measures). Review of literature suggests a very high incidence of gain of chromosome 8 with PPB. ${ }^{23}$ Interestingly, association of gain of chromosome 8 is also reported in a high proportion of cases of ERMS and some cases of mesenchymal chondrosarcomas. ${ }^{24,25}$ This molecular association has not yet been well explored in DICER1 -related sarcomas, needs further investigation. A recent landmark paper of a DNA methylation based sarcoma classifier found that DICER1 mutated sarcomas cluster independent (methylation class sarcoma RMS-like) from conventional embryonal and MYOD1 mutated rhabdomyosarcomas and may represent a distinct group of tumours. This distinction is further sharpened as these tumours do not respond to standard RMS protocol chemotherapy. All the DICER1 mutated sarcomas in their cohort showed gain of chromosome 8 as well. ${ }^{26}$

Treatment guidelines are still not standard for these tumours, though most reports have used an anthracycline and alkylator based protocol with varied response. ${ }^{8}$ These tumours seem to benefit from initially a more intensive chemotherapy backbone followed by a prolonged maintenance chemotherapy strategy, in spite of 
the presence of RMS differentiation, thus making the accurate diagnosis of DICER1 mutated sarcoma, vital. All our patients had aggressive disease but responded to the alternating VDC/PEI backbone followed by maintenance chemotherapy. The decision for VDC/PEI for the CNS case was based on a multidisciplinary tumor board review with expert consensus from around the world and then it became our standard for the subsequent tumors in view of the excellent responses observed serially. Satisfactory local control with surgery and/or radiotherapy seems imperative for maintaining remission. Prognosis can be favorable, provided the tumours are detected early. ${ }^{1,2,27}$

The risk of developing a tumour associated with germline DICER1 variation is greatest in early childhood $\left(1^{\text {st }}\right.$ decade $)$ and decreases with increasing age and surveillance protocols are developed accordingly. Correct and early diagnosis of DICER1syndrome is imperative to ensure appropriate surveillance protocols are followed in these patients for improving survival. Apart from macrocephaly in some individuals, this cancer predisposition syndrome is typically not associated with specific phenotypic characteristics, making clinical identification difficult. Hence histologic identification is the key and the onus rests on the pathologist to correctly identify the morphologic clues to guide testing forDICER1 mutation, especially in resource constrained settings. The presence of rhabdomyoblastic differentiation along with primitive blastemal and mesenchymal component, with or without chondroid differentiation are the key features which should raise suspicion, especially in pediatric sarcomas of the female genitourinary tract or brain.

Disclosure / Conflict of interest : The authors D. Ashley Hill and KrisAnn Schultz are supported by National Institutes of Health grant NCI R01CA143167 and NCI R37CA24494. The International PPB/DICER1Registry gratefully acknowledges philanthropic support from the Pine Tree Apple Classic Fund and Children's Minnesota Foundation.

The other authors have no conflict of interest and nothing to disclose.

Ethical approval : Institutional review board approval ((EC/WV/TMC/41/20) has been taken for this study.

Informed consent - Informed consent was taken from patients' parents before this study

Funding - None. Testing for DICER1 was supported by the DICER1/ Pleuro-Pulmonary Blastoma society. Courier charges were borne by Tata Medical Center.

\section{Acknowledgements}

Dr Stefan Rutkowski, Dr Jonas Ecker, Dr Kerstin Grund and The Pediatric Targeted Therapy 2.0 program at the German Cancer Reasearch Center (DKFZ), Heidelberg University

\section{References :}

1. Stewart DR, Best AF, Williams GM, et al. Neoplasm Risk Among Individuals With a Pathogenic Germline Variant in DICER1. J Clin Oncol. 2019;37(8):668-676.

2. Schultz KAP, Williams GM, Kamihara J, et al. DICER1 and Associated Conditions: Identification of At-risk Individuals and Recommended Surveillance Strategies. Clin Cancer Res. 2018;24(10):2251-2261.

3. Robertson JC, Jorcyk CL, Oxford JT. DICER1 Syndrome: DICER1 Mutations in Rare Cancers. Cancers (Basel). 2018;10(5).

4. Kim J, Schultz KAP, Hill DA, Stewart DR. The prevalence of germline DICER1 pathogenic variation in cancer populations. Mol Genet Genomic Med. 2019;7(3):e555.

5. de Kock L, Wu MK, Foulkes WD. Ten years of DICER1 mutations: Provenance, distribution, and associated phenotypes. Hum Mutat.2019;40(11):1939-1953.

6. Schultz KA, Yang J, Doros L, et al. DICER1-pleuropulmonary blastoma familial tumor predisposition syndrome: a unique constellation of neoplastic conditions. Pathol Case Rev. 2014;19(2):90-100. 
7. Warren M, Hiemenz MC, Schmidt R, et al. Expanding the spectrum of dicer1-associated sarcomas. Mod Pathol. 2020;33(1):164-174.

8. Schultz KAP, Nelson A, Harris AK, et al. Pleuropulmonary blastoma-like peritoneal sarcoma: a newly described malignancy associated with biallelic DICER1 pathogenic variation. Mod Pathol. 2020;33(10):19221929.

9. Nakano Y, Hasegawa D, Stewart DR, et al. Presacral malignant teratoid neoplasm in association with pathogenic DICER1 variation. Mod Pathol. 2019;32(12):1744-1750.

10. McCluggage WG, Foulkes WD. DICER1-associated sarcomas: towards a unified nomenclature. Mod Pathol. 2020.

11. Pancaldi A, Peng L, Rhee DS, et al. DICER1-associated metastatic abdominopelvic primitive neuroectodermal tumor with an EWSR1 rearrangement in a 16-yr-old female. Cold Spring Harb Mol Case Stud. $2020 ; 6(5)$.

12. McCluggage WG, Apellaniz-Ruiz M, Chong AL, et al. Embryonal Rhabdomyosarcoma of the Ovary and Fallopian Tube: Rare Neoplasms Associated With Germline and Somatic DICER1 Mutations. Am J Surg Pathol. 2020;44(6):738-747.

13. de Kock L, Priest JR, Foulkes WD, Alexandrescu S. An update on the central nervous system manifestations of DICER1 syndrome. Acta Neuropathol. 2020;139(4):689-701.

14. Apellaniz-Ruiz M, Cullinan N, Grant R, et al. DICER1 screening in 15 paediatric paratesticular sarcomas unveils an unusual DICER1-associated sarcoma. J Pathol Clin Res. 2020;6(3):185-194.

15. Doros LA, Rossi CT, Yang J, et al. DICER1 mutations in childhood cystic nephroma and its relationship to DICER1-renal sarcoma. Mod Pathol. 2014;27(9):1267-1280.

16. Yoon JY, Apellaniz-Ruiz M, Chong AL, et al. The Value of DICER1 Mutation Analysis in "Subtle" Diagnostically Challenging Embryonal Rhabdomyosarcomas of the Uterine Cervix. Int J Gynecol Pathol.2020.

17. Doros L, Yang J, Dehner L, et al. DICER1 mutations in embryonal rhabdomyosarcomas from children with and without familial PPB-tumor predisposition syndrome. Pediatr Blood Cancer.2012;59(3):558-560.

18. McCluggage WG, Chong AL, de Kock L, Foulkes WD. Somatic tumour testing establishes that bilateral DICER1-associated ovarian Sertoli-Leydig cell tumours represent independent primary neoplasms. Histopathology. 2020.

19. Apellaniz-Ruiz M, McCluggage WG, Foulkes WD. DICER1-associated embryonal rhabdomyosarcoma and adenosarcoma of the gynecologic tract: Pathology, molecular genetics, and indications for molecular testing. Genes Chromosomes Cancer. 2021;60(3):217-233.

20. de Kock L, Yoon JY, Apellaniz-Ruiz M, et al. Significantly greater prevalence of DICER1 alterations in uterine embryonal rhabdomyosarcoma compared to adenosarcoma. Mod Pathol. 2020;33(6):1207-1219.

21. Kamihara J, Paulson V, Breen MA, et al. DICER1-associated central nervous system sarcoma in children: comprehensive clinicopathologic and genetic analysis of a newly described rare tumor. Mod Pathol.2020;33(10):1910-1921.

22. Koelsche C, Mynarek M, Schrimpf D, et al. Primary intracranial spindle cell sarcoma with rhabdomyosarcoma-like features share a highly distinct methylation profile and DICER1 mutations. Acta Neuropathol. 2018;136(2):327-337.

23. Mlika M, Anjum F, El Mezni F. Pleuropulmonary Blastoma. In:StatPearls. Treasure Island (FL)2021.

24. Shern JF, Chen L, Chmielecki J, et al. Comprehensive genomic analysis of rhabdomyosarcoma reveals a landscape of alterations affecting a common genetic axis in fusion-positive and fusion-negative tumors. Cancer Discov. 2014;4(2):216-231. 
25. Arora K, Riddle ND. Extraskeletal Mesenchymal Chondrosarcoma.Arch Pathol Lab Med. 2018;142(11):1421-1424.

26. Koelsche C, Schrimpf D, Stichel D, et al. Sarcoma classification by DNA methylation profiling. Nat Commun. 2021;12(1):498.

27. Schultz KA, Harris A, Williams GM, et al. Judicious DICER1 testing and surveillance imaging facilitates early diagnosis and cure of pleuropulmonary blastoma. Pediatr Blood Cancer.2014;61(9):1695-1697.

\section{Figure legends :}

Fig 1.

a. MRI showing - Sagittal T2W MRI image shows an intra-axial large solid -cystic mass measuring $7 \mathrm{~cm} \mathrm{x}$ $6 \mathrm{~cm}$ in the right parafalcine fronto-parietal lobe, causing ventricular compression. (Case 1)

b. Contrast enhanced axial CT image multiple lobulated bilateral adnexal lesions and lesions in the pouch of Douglas (asterisk). (Case 2)

c. Post-treatment axial contrast enhanced CT image showing significant reduction in size and extent of the lesions (asterisk). (Case 2)

d. Residual intermediate T2W signal intensity lesion in the endocervical canal (asterisk). (Case 3)

e-f. Subsequent T2W sagittal images show increasing size of the recurrent lesion at the same site (asterick). (Case 3)

Fig 2. $\mathrm{H} \& \mathrm{E}$

a. Scanner view of the immature cartilage, $\mathrm{x} 2.5$ magnification.

b. Scanner view of the malignant primitive blastemal like small spindle cells, $\mathrm{x} 4$ magnification.

c. Low power of the subepithelial arrangement of primitive blastemal like spindle cells x 10 magnification.

d. Low power of the tumour cells showing hypocellular and hypercellular areas x 10 magnification. Immunohistochemistry with desmin showing positivity in the spindle cells (inset).

e. Low power view of the immature cellular cartilage admixed with the spindle cells, $\mathrm{x} 10$ magnification.

f. High power view showing abrupt transition from chondroid to spindle cells, x 40 magnification.

Fig 3. $\mathrm{H} \& \mathrm{E}$

a. Scanner view showing the immature cartilage and malignant spindle cells, $\mathrm{x} 4$ magnification.

b. Low power view showing alternating hypercellular and hypocellular areas in a myxoid background, x 10 magnification. The spindle cells are positive for desmin (inset).

c. Low power view showing subepithelial condensation of tumour cells, x 10 magnification.

d. Low power view showing densely packed primitive blastemal cells showing perivascular condensation of tumour cells, x 10 magnification.

e. Low power view showing tumour cells in nests in a loose myxoid background, x 10 magnification.

f. High power view of the tumour cells showing coarse chromatin and mitotic figures, x 40 magnification.

Fig 4.

a. Scanner view of primary tumour showing the mixed pattern, comprising of hypocellular and hyper cellular areas, x 2.5 magnification. 
b. Low power view showing subepithelial concentration of spindle cells, mimicking a cambium-like layer, $\mathrm{x}$ 10 magnification. The spindle shaped tumour cells are strongly positive for desmin (inset).

c. Low power view showing immature mesenchyme, x 20 magnification.

d. Low power view showing hypercellular foci of larger cells in nests, cords and showing mitotic figures, $\mathrm{x}$ 10 magnification.

e. Low power view showing granular pink neuropil / glia-like area with rosette formation in the tumour, $\mathrm{x}$ 10 magnification.

f. Low power view showing tumour cells arranged in cords, x 20 magnification.

g. Low power of the biopsy from residual tumour showing scattered bland spindle cells in a loose myxoid background, x 10 magnification.

h. Low power view of recurrent tumour showing sheets of large blastemal cells x 10 magnification.

i. High power view of the blastemal cells showing opened up nuclear chromatin and mitoses and apoptosis, x 40 magnification. The blastemal cells are strongly positive for SALL4 (inset).

\section{Hosted file}

Table.docx available at https://authorea.com/users/418471/articles/525210-phenotypicsimilarities-within-the-morphologic-spectrum-of-dicer1-associated-sarcomas-andpleuropulmonary-blastoma-histopathologic-features-suggesting-genetic-testing-andcounselling
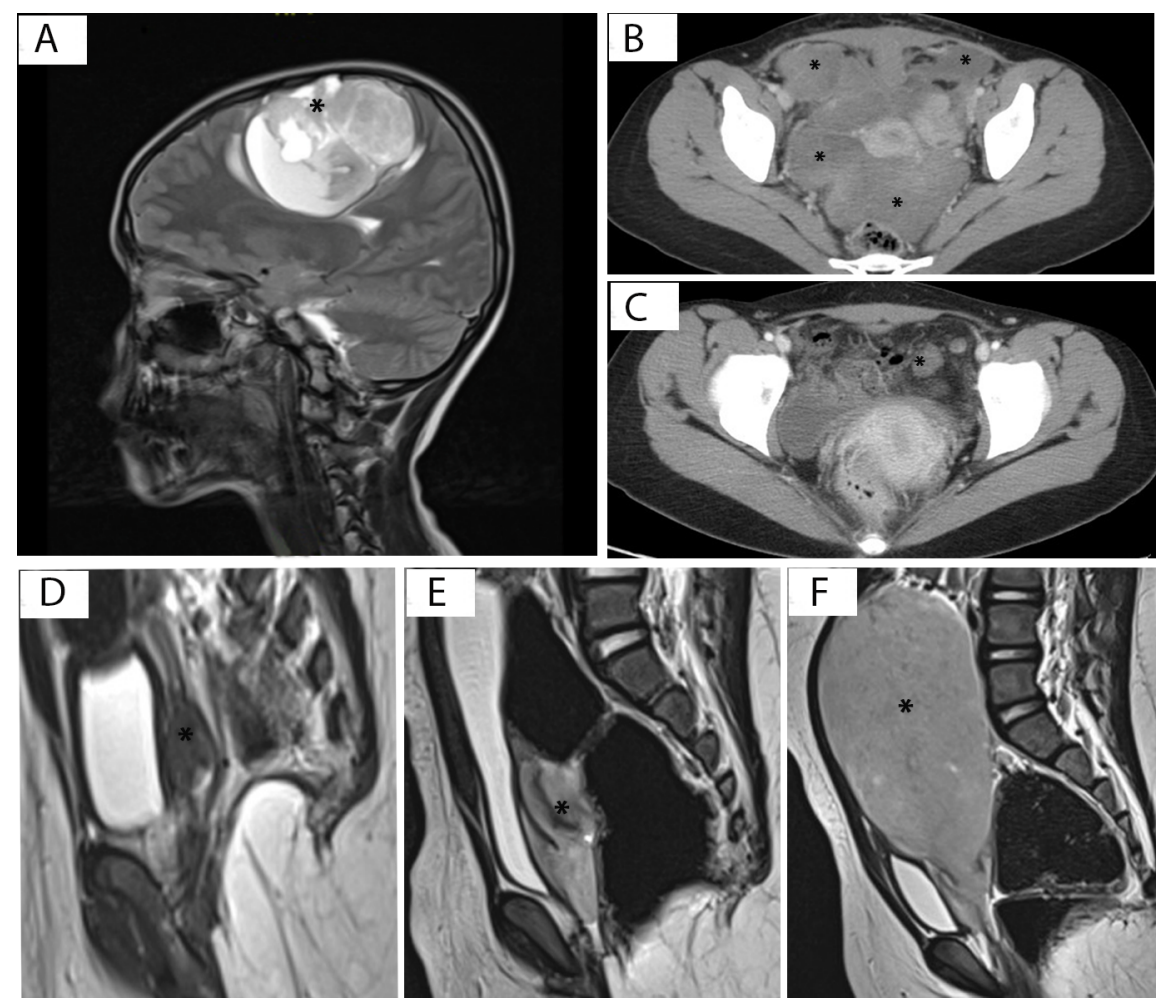

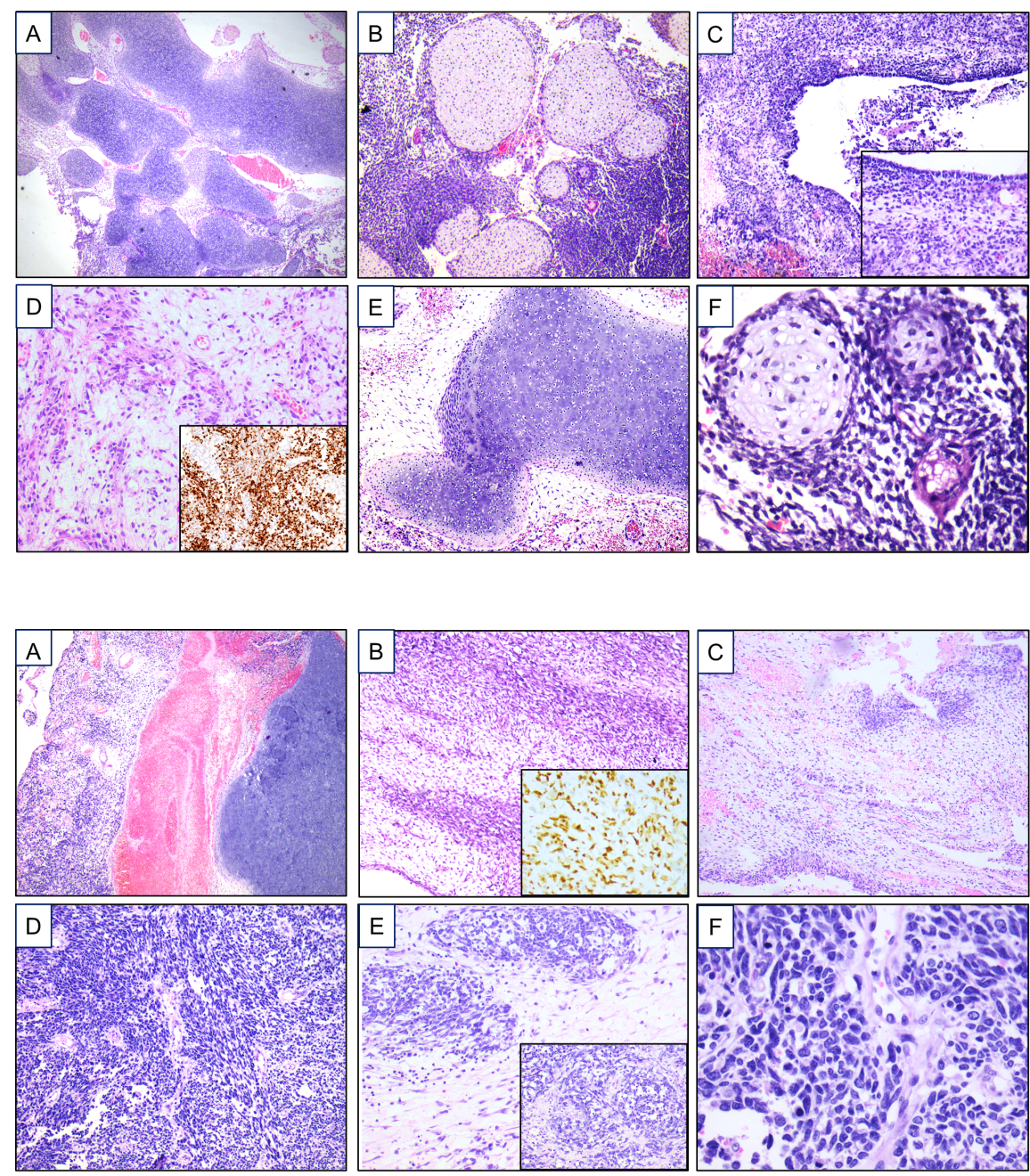

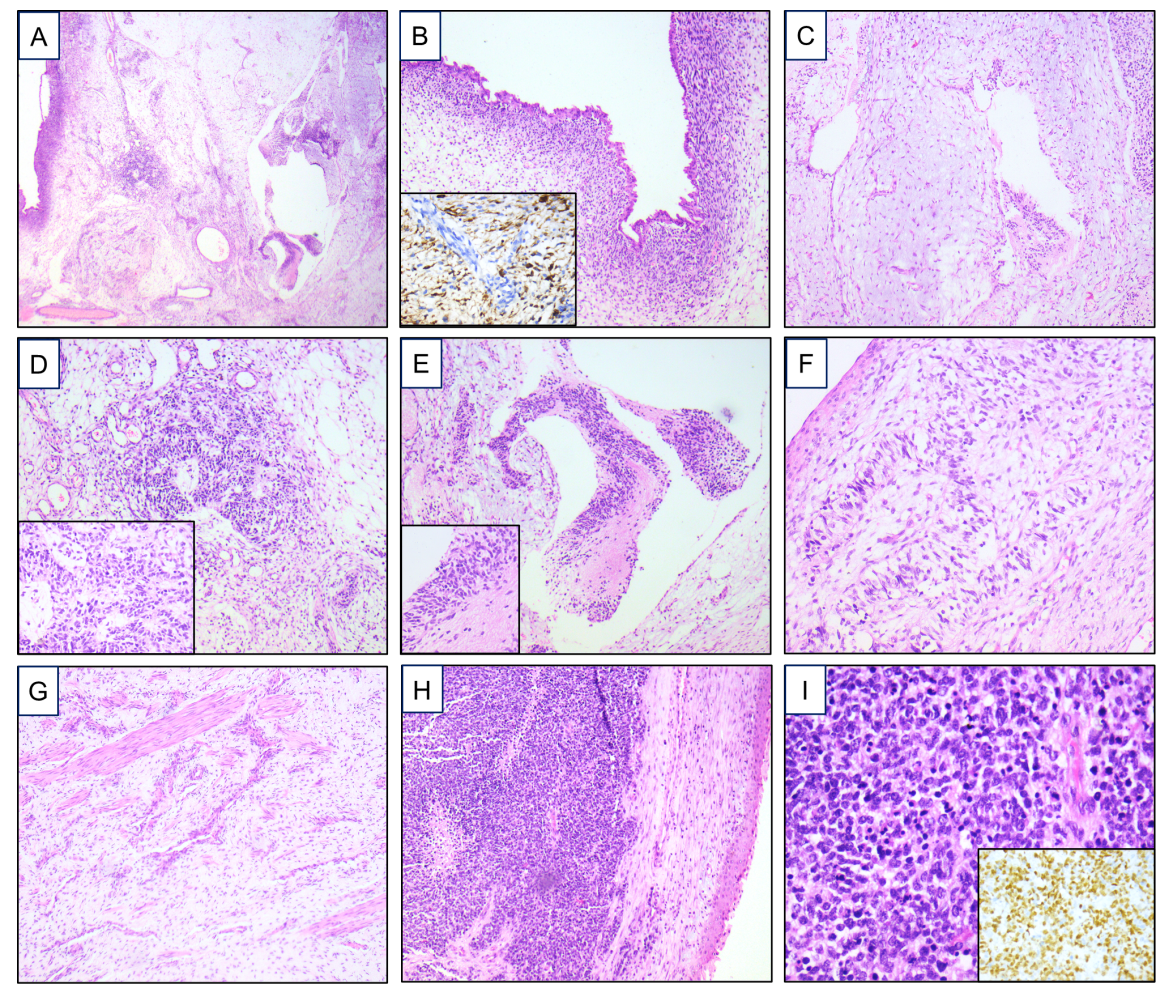